\title{
Evidence-Based Imaging in Pediatrics
}

\author{
L.S. Medina, K.E. Applegate, and C.C. Blackmore, eds.
}

New York, NY: Springer, 2010, 665 pages. $\$ 79.95$

This is a book that will appeal to an audience of radiologists, pediatricians, primary care physicians, residents and fellows, midlevel providers, and others in the fields of diagnostic radiology and pediatric radiology who request imaging for children. Through a review of the literature and an algorithmic approach, the authors provide a guide to efficient imaging of specific clinical problems in children while minimizing unnecessary radiation risk and efficiently using health care dollars. A total of 41 chapters are included, with sections on neuroimaging, the musculoskeletal system, the chest, the abdomen, and prenatal imaging.

The first 3 chapters address the principles of evidencebased imaging, a critical assessment of the literature with regard to error and bias, and radiation risk from medical imaging in children. Other chapters address common issues in pediatric imaging such as nonaccidental trauma, neuroimaging of seizures, mediastinal masses, congenital heart disease, developmental dysplasia of the hip, and urinary tract infection.

Each chapter opens with specific issues and key points to address them. Each clinical entity is further described with definition and pathophysiology, epidemiology, overall cost to society, clinical and laboratory predictors, goals of imaging, and the methodology used in the review of the literature. The key imaging issues are then further discussed, with citations. Imaging algorithms are included where appropriate, and questions for further research are provided. At the end of each chapter are imaging case studies with classic imaging findings, followed by references. Using this format, the book often concisely answers the reader's question in the well-written initial paragraphs, rather than leaving one to complete the entire chapter and summarize on one's own. Each chapter, although not long (usually $12-15$ pages, with 30 pages being the longest), is packed with highly useful information. Many chapters include imaging protocols.

Limitations of the book include its lack of detail on the topics of neck, liver, and biliary tract imaging. Suggestions for the next edition include chapters on complications of solid-organ and stem cell transplantations and on imaging for pediatric hypertension.

I expected to be overwhelmed by the book but found it enjoyable. There was no such reference 10 years ago, after the panic of late January 2001 initiated by lay press articles regarding pediatric CT dose and cancer. Pediatric radiology as a specialty responded by developing more thoughtful processes and better imaging methods, many of which are summarized in this commendable book.

\section{Nancy E. Fitzgerald}

M.D. Anderson Cancer Center

1515 Holcombe Blvd., Unit 368

Houston, TX 77030

E-mail: nancy.fitzgerald@di.mdacc.tmc.edu 